ARTIGO DE REVISÃO REVIEW ARTICLE

\section{Custo do erro de medicação e eventos adversos à medicação na cadeia medicamentosa: uma revisão integrativa}

\author{
Cost of the medication error and adverse drug events in the \\ medication therapy chain: review literature as a topic
}

Renata Prado Bereta Vilela', Daniele Alcalá Pompeo², Marli de Carvalho Jericó², Alexandre Lins Werneck³

DOI: $10.21115 / J B E S . v 10 . n 2 . p 179-189$

\begin{abstract}
RESUMO
Objetivo: Analisar os estudos sobre o custo dos erros de medicação e eventos adversos à medicação nas etapas da cadeia medicamentosa. Métodos: Revisão integrativa, realizada nas bases de dados LILACS (Literatura Latino-Americana e do Caribe em Ciências da Saúde), CINAHL (Cumulative Index to Nursing and Allied Health Literature) e MEDLINE (Medical Literature Analysis and Retrieval Sistem on-line), no período de 2005 a 2017. Resultados: Foram analisados 19 artigos, com predomínio do nível 6 de evidência, tanto pelo sistema hierárquico $(63,2 \%)$ quanto para avaliações econômicas $(42,1 \%)$. Dentre os estudos, sete estavam relacionados à prescrição, um, à administração, dois, à prescrição e administração e nove contemplavam todas as etapas da cadeia medicamentosa. Houve grande assimetria de valores apresentada na estimação de custo evitável/erro de medicação, com média de US\$ 617.493.770,36, variando de US\$ 83,32 a US\$ 5.095.640.000,00. Conclusão: Foi possível comprovar que os erros de medicação podem gerar alto custo e representam uma importante fonte de desperdício e ineficiência hospitalar, reiterando a importância na adoção de medidas preventivas.
\end{abstract}

\section{Keywords:}

medication errors, costs and cost analysis, patient safety, medication systems, hospital, nursing

\begin{abstract}
Objective: Analyze the scientific production related to the cost of medication errors and adverse drug events in the stages of the medication therapy chain. Methods: We conducted an integrative review of the published literature from 2005 to 2017. Databases searched included LILACS (Latin American and Caribbean Center on Health Sciences Information), CINAHL (Cumulative Index to Nursing and Allied Health Literature), and MEDLINE (Medical Literature Analysis and Retrieval System on-line). Results: We analyzed 19 articles with a predominance of evidence level 6 through both the hierarchical system (63.2\%) and economic evaluations (42.1\%). Among the articles, seven were related to drug prescription, one to medication therapy management, two were related to prescription and medication therapy management, and nine included all stages of the medication therapy chain. There was a great mismatch of values presented in the estimation of avoidable cost/medication error, with a mean cost of US\$ 617.493.770,36, ranging from US\$ 83,32 to US\$ 5,095,640,000.00. Conclusion: We concluded that it was possible to prove that medication errors can generate high costs and they represent an important source of medical waste and hospital inefficiency. Thus, we stress the importance of adopting preventive measures.
\end{abstract}

Recebido em: 13/06/2018. Aprovado para publicação em: 03/07/2018.

1. Mestre, Docente do Curso de Medicina da Faceres, São José do Rio Preto, SP, Brasil.

2. Professora Doutora, Departamento de Enfermagem Especializada, Faculdade de Medicina de São José do Rio Preto, São José do Rio Preto, SP, Brasil.

3. Professor Doutor, Departamento de Ciências da Saúde, Faculdade de Medicina de São José do Rio Preto, São José do Rio Preto, SP, Brasil.

Manuscrito extraído da dissertação de mestrado intitulada "Erro de medicação: o custo e o impacto das tecnologias preventivas na cadeia medicamentosa", apresentada em 2016, na Faculdade de Medicina de São José do Rio Preto.

Fontes de financiamento: Este trabalho foi desenvolvido com recursos próprios dos autores, não havendo nenhum financiamento, equipamento nem medicamento concedidos por terceiros.

Trabalho apresentado na forma de pôster no XIII Encontro de Enfermeiros de Hospitais de Ensino do Estado de São Paulo, no período de 20 a 22 de setembro de 2016, na Universidade de Campinas (Unicamp), Campinas, SP.

Autor correspondente: Renata Prado Bereta Vilela. Av. Anísio Hadad, 6751, Jd. São Francisco, São José do Rio Preto, SP. CEP: 15090305. Telefone: (17) 99713-3799. E-mail: renata_bereta@hotmail.com 


\section{Introdução}

O processo de fornecimento de medicação intra-hospitalar, também denominado cadeia medicamentosa, é multidisciplinar e normalmente composto de três etapas (prescrição, dispensação e administração de medicamentos), variando de acordo com a instituição de saúde. A prescrição é realizada pelo médico e, se houver, transcrição, é realizada pelo escriturário ou enfermeiro. Essa etapa pode não existir em instituições que utilizam a prescrição eletrônica, uma vez que esta é enviada automaticamente para a farmácia. A dispensação é realizada pela equipe da farmácia e a administração de medicamentos é frequentemente realizada pela equipe de enfermagem. Dessa forma, o erro de medicação pode ocorrer em qualquer etapa desse processo (Pazin Filho et al., 2013).

O erro de medicação é definido como qualquer evento evitável que ocorra em qualquer fase da terapia medicamentosa, podendo ou não a prescrição, a dispensação ou a administração causar algum dano ao paciente (Cassiani et al., 2010; Belela et al., 2011). Já os eventos adversos a medicamentos (EAM) são "qualquer dano ou lesão causada ao paciente pela intervenção médica relacionada aos medicamentos". Os EAM podem ser divididos em preveníveis e não preveníveis; os preveníveis, ou seja, que podem ser prevenidos pela equipe de saúde, são considerados erros de medicação graves (Anacleto et al., 2010; Kane-Gill et al., 2010).

Tanto os EAM quanto os erros de medicação podem aumentar o tempo de permanência dos pacientes nas instituições de saúde, o que está intimamente associado ao aumento de custos (Ucha Samartin et al., 2013). Em 2011, um estudo que estimou o custo dos erros de medicação de quimioterápicos relatou que os 436 erros interceptados poderiam gerar 216 dias adicionais de hospitalização e um custo evitável/ano de US\$126,353.52 (Ranchon et al., 2011).

A questão da segurança do paciente está muito presente na atualidade, em virtude de seu grande impacto econômico para a saúde. No entanto, pesquisas que abordam os custos do erro de medicação ainda são escassas. Um estudo relevante, realizado em 1997, que objetivou avaliar a utilização do recurso adicional associado aos EAM, apresentou um potencial de custo de US\$ 5,6 milhões/ano, sendo US\$2,595.00/ evento e de eventos preveníveis, US\$ 4,685.00 (Bates et al., 1997). Essa temática ganhou mais destaque em 1999 com a publicação do relatório americano To err is human: building a safer health system, que estimou a morte de 44 mil a 98 mil pessoas em decorrência de erros médicos que poderiam ser prevenidos, representando um potencial custo de US\$ 2,8 milhões ao ano (Kohn et al., 1999).

Entende-se que é fundamental analisar na literatura o custo do erro de medicação e EAM relacionado às etapas de prescrição, dispensação e administração de medicamentos, a fim de contribuir para uma prática mais segura ao paciente, ao profissional e à instituição, além de estimular investigações futuras sobre essa temática que se apresenta escassa na literatura. Dessa forma, esta pesquisa objetivou analisar os estudos sobre o custo dos erros de medicação e eventos adversos à medicação nas etapas da cadeia medicamentosa.

\section{Métodos}

Para a elaboração desta revisão integrativa, foram seguidas estas etapas: identificação da questão da pesquisa, busca na literatura, categorização e avaliação dos estudos, interpretação dos resultados e síntese do conhecimento (Whittemore et al., 2014). A questão norteadora consistiu em "O custo do erro de medicação e EAM tem sido investigado na literatura em qual etapa da cadeia medicamentosa?".

A busca dos estudos foi realizada nas bases de dados: LILACS (Literatura Latino-Americana e do Caribe em Ciências da Saúde), que é o mais importante e abrangente índice da literatura científica e técnica da América Latina e Caribe; $\mathrm{Cl}$ NAHL (Cumulative Index to Nursing and Allied Health Literature), sistema de informação internacional, exclusivo da EBSCO (Elton B. Stephens Company), de literatura voltada à área da saúde, especificamente da enfermagem, e MEDLINE (Medical Literature Analysis and Retrieval Sistem on-line), acessada por meio do sistema PubMed, banco desenvolvido e mantido pela Biblioteca Nacional de Medicina dos Estados Unidos.

Estabeleceram-se como critérios de inclusão artigos nos idiomas português, inglês ou espanhol, limitados a humanos e publicados no período de 2005 a 2017. Optou-se por esse período para que os dados estivessem mais próximos à atualidade, facilitando comparações práticas.

A busca foi realizada no período de 10 de janeiro de 2015 a 5 de janeiro de 2018 por dois pesquisadores de forma independente (índice de concordância: 100\%). Para a busca, foram utilizados os descritores controlados da Biblioteca Regional de Medicina (BIREME), Descritores em Ciências da Saúde (DECS) e da National Library of Medicine (NLM), Medical Subjects Headings (MeSH), combinados por meio do operador booleano AND: "Erros de Medicação" e "Custos e análise de custo" e "Medication Errors" e "Costs and Cost Analysis".

Foram identificados 407 artigos (PubMed: 375; CINAHL: 32; Lilacs: 0), tendo sido excluídos 388. Inicialmente, foi realizada a leitura do título e resumo da publicação para verificar o atendimento aos critérios de inclusão. Nos casos em que o título e o resumo não foram suficientes para definir a temática pesquisada, buscou-se a publicação na íntegra, de forma que todos os critérios pudessem ser aplicados e os artigos que respondessem à questão norteadora do estudo fossem selecionados. O Quadro 1 mostra a busca, inclusão e exclusão dos artigos analisados.

Para extrair os dados dos artigos incluídos nessa revisão, utilizou-se instrumento de coleta de dados contendo variáveis 
relacionadas a identificação do estudo, introdução e objetivo, características metodológicas, resultados e conclusões.

O nível de evidência foi avaliado por meio de duas ferramentas. A primeira ocorreu por meio do sistema hierárquico científico (Melnyk \& Fineout Overhold, 2011), composto de sete níveis: nível 1 (revisões sistemáticas com ou sem metanálise de ensaios clínicos randomizados e guidelines baseados em revisão sistemática de ensaios clínicos randomizados); nível 2 (pelo menos um ensaio clínico randomizado); nível 3 (ensaios clínicos sem randomização); nível 4 (estudos com caso controle e de coorte); nível 5 (revisões sistemáticas de estudos descritivos ou qualitativos); nível 6 (um único estudo quantitativo ou qualitativo) e nível 7 (opinião de autoridade ou comitê de especialistas).

A segunda ferramenta utilizada foi um instrumento específico para avaliar estudos que incluiu a temática custos, denominado Levels of Evidence for Economic Evaluation (Joanna Briggs Institute, 2013), composto de sete níveis: nível 1 (modelo de decisão baseado em revisão sistemática realizado no local de tomada de decisão); nível 2 (revisão sistemática de avaliações econômicas conduzidas em local similar ao de tomada de decisão); nível 3 (síntese ou revisão de avaliação econômica realizada em uma configuração semelhante à de tomada de decisão, feitas com alta qualidade; nível 4 (avaliação econômica de alta qualidade); nível 5 (síntese ou revisão de avaliação econômica de moderada e/ou pobre qualidade); nível 6 (avaliação econômica simples de moderada ou pobre qualidade); nível 7 (opinião de especialistas sobre custos de intervenções e comparações).

Nos estudos de economia da saúde, os custos são divididos nas categorias: diretos, ou seja, aqueles diretamente relacionados aos serviços de saúde que implicam dispêndios imediatos; indiretos, aqueles relativos às mudanças da capacidade produtiva do indivíduo e familiares decorrentes do processo de adoecimento ou mortalidade precoce; e intangíveis, os de difícil mensuração monetária, como a dor, o sofrimento, entre outros (Tonon et al., 2008).

Para facilitar a compreensão do custo nas etapas da cadeia medicamentosa, optou-se por uma padronização da moeda, o dólar, em razão de sua ampla utilização, realizando-se a conversão do valor de origem para o dólar, com referência ao valor do ano de publicação do estudo.

A apresentação dos resultados foi realizada por meio de um quadro sinóptico contendo informações-chave dos estudos primários e de forma descritiva, de acordo com a etapa da cadeia medicamentosa, o nível de evidência das pesquisas e o delineamento do estudo.

\section{Resultados}

No presente estudo, foram analisados 19 artigos que atenderam aos critérios de inclusão previamente estabelecidos,

tendo sido apresentado um panorama geral das pesquisas avaliadas.

Em relação ao ano de publicação, um foi publicado em 2005, dois, em 2007, um, em 2008, dois, em 2009, dois, em 2011, três, em 2012, três, em 2013, dois, em 2014, um, em 2016 e dois, em 2017. Desses artigos, dez foram norte-americanos, dois, franceses, dois, espanhóis, um, suíço, um, mexicano, um, chinês, um, irlandês e um, iraniano; sendo três deles publicados no idioma espanhol e os demais em inglês. Não houve trabalhos nacionais incluídos na amostra. Participaram dos estudos 93 autores, dos quais 39 (41,9\%) eram farmacêuticos, 27 (29,1\%), médicos, três (3,2\%), enfermeiros, três $(3,2 \%)$, economistas, dois $(2,2 \%)$, estatísticos e $19(20,4 \%)$ autores dos quais não foi possível identificar a categoria profissional.

No que diz respeito ao nível de evidência, houve predomínio do nível 6 tanto pelo sistema de hierarquia científico (12; 63,2\%) quanto para a análise econômica (8; 42,1\%), com delineamento descritivo com relação aos objetivos em 11 $(57,9 \%)$ e adoção de estudo de caso como procedimento técnico em dez $(52,6 \%)$ estudos. Fizeram parte da amostra duas $(10,5 \%)$ revisões sistemáticas.

Quanto à categoria do tipo de custo (direto, indireto e intangível) das avaliações econômicas analisadas, encontraram-se 16 (84,2\%) diretos, um (5,3\%) indireto e dois (10,5\%) eram revisões sistemáticas que utilizaram vários estudos e não especificaram o método de custeio utilizado. Por fim, não houve estudo envolvendo custo intangível.

Entre as etapas da cadeia medicamentosa, sete estudos (36,8\%) abordaram apenas a prescrição médica (Ucha Samartin, 2013; Lampert et al., 2008; Aldrid et al., 2009; Nerich et al., 2012; Kopp et al., 2007; Charles et al., 2014) (Quadro 1). Não

Quadro 1. Figura de identificação, exclusão e seleção dos artigos. São José do Rio Preto, SP, Brasil, 2018

\section{BASES DE DADOS \\ LILACS: Erros de Medicação AND Custos \\ e Análise de Custo $=0$ estudo \\ PubMed: Medication Errors AND Costs and Cost Analysis $=375$ estudos \\ CINAHL: Medication Errors AND Costs and Cost Analysis $=32$ estudos}

Critérios de inclusão: artigos envolvendo seres humanos, nos idiomas português, inglês ou espanhol, publicados entre 2005 e 2017.

\section{PubMed $=18$}

Exclusões ( $n=357)$ : período $(n=119)$; idioma $(n=16)$; realizados em animais $(n=$ 7); duplicidade $(n=1)$; não relacionado ao tema $(n=214)$

\section{AMOSTRA $=19$ estudos}

Fonte: Elaborado pelo autor.

\section{CINAHL $=1$}

Exclusões $(n=31)$ : período $(n=12)$, não relacionado ao tema $(n=19)$ 
houve pesquisa que discutiu exclusivamente a dispensação e somente um (5,3\%) artigo focalizou a administração de medicamentos (Letvak et al., 2012) (Quadro 2). Dentre os estudos que se referiram a mais de uma etapa da cadeia medicamentosa (Quadro 3), dois (10,5\%) incluíram prescrição médica e administração de medicamentos (Lada \& Delgado Junior,
2007; Gharekhani et al., 2014). Por fim, nove $(47,4 \%)$ pesquisas abarcaram as três etapas da cadeia medicamentosa (prescrição, dispensação e administração) (Ranchon et al., 2011; Aceves Avila et al., 2011; Paradis et al., 2009; Hug et al., 2012; Cohen et al., 2005; Schneider, 2013; Villar, 2013; Walsh et al., 2017; Choi et al., 2016).

Quadro 2. Distribuição da análise dos estudos sobre o custo dos erros de medicação e evento adverso à medicação em apenas uma etapa da cadeia medicamentosa. São José do Rio Preto, SP, Brasil, 2018

\begin{tabular}{|c|c|c|c|}
\hline $\begin{array}{l}\text { Cadeia } \\
\text { medicamentosa e *NE }\end{array}$ & Objetivo & Método & Resultado \\
\hline $\begin{array}{l}\text { †P } \\
\text { Evidência: } 6 \\
\text { †AE: } 5 \\
\text { País: Suíça } \\
\text { (Lampert et al., 2008) }\end{array}$ & $\begin{array}{l}\text { Avaliar as intervenções } \\
\text { farmacêuticas relacionadas } \\
\text { à redução do uso de } \\
\text { medicamentos por meio de um } \\
\text { software da rede europeia }\end{array}$ & $\begin{array}{l}\text { Delineamento: descritivo - } \\
\text { Estudo de caso } \\
\text { Tipo de custo: direto - } \\
\text { somatório dos custos dos } \\
\text { medicamentos economizados } \\
\text { Tipo de evento: erro de medicação }\end{array}$ & $\begin{array}{l}\text { Pacientes: } 1.444 \\
\text { Intervenções: } 213 \\
\text { §EP: } 51 \\
\text { Custo evitável/ano: US\$ 12.555,27 }\end{array}$ \\
\hline $\begin{array}{l}\text { †P } \\
\text { Evidência: } 6 \\
\text { ¥AE: } 5 \\
\text { País: Estados Unidos } \\
\text { (Aldrid et al., 2009) }\end{array}$ & $\begin{array}{l}\text { Descrever a implantação de um } \\
\text { software para revisão de prescrições } \\
\text { e identificação do evento, } \\
\text { classificação e estimar o custo } \\
\text { evitável em unidade de emergência }\end{array}$ & $\begin{array}{l}\text { Delineamento: } \\
\text { descritivo - } \\
\text { Estudo de caso } \\
\text { Tipo de custo: direto - projeção } \\
\text { de custo evitável - não } \\
\text { menciona como foi calculado, } \\
\text { mas cita Lee et al. (2002) } \\
\text { Tipo de evento: erro de medicação }\end{array}$ & $\begin{array}{l}\text { Intervenções: } 9.568 \\
\text { §EP: } 668 \text { (prevenidos) } \\
\text { Custo evitável/ano: US\$ 1.691.185,00 }\end{array}$ \\
\hline $\begin{array}{l}\text { †P } \\
\text { Evidência: } 4 \\
\text { łAE: } 5 \\
\text { País: França } \\
\text { (Nerich et al., 2012) }\end{array}$ & $\begin{array}{l}\text { Comparar o não uso com o uso } \\
\text { de computerized physician order } \\
\text { entry (CPOE) para prevenir erro } \\
\text { de medicação em oncologia }\end{array}$ & $\begin{array}{l}\text { Delineamento: descritivo - } \\
\text { Estudo de caso } \\
\text { Análise do custo-benefício } \\
\text { impedindo §EP } \\
\text { Tipo de custo: direto - Sistema de } \\
\text { financiamento de saúde francês } \\
\text { Tipo de evento: erro de medicação }\end{array}$ & $\begin{array}{l}\text { Prescrições: } 14.854 \\
\text { Intervenções: } 459 \\
\text { §EP: } 218 \\
\text { Custo da análise farmacêutica: } \\
\text { US\$ } 25.0618,00 \\
\text { Custo evitável/ano: US\$ 372.267,56 }\end{array}$ \\
\hline $\begin{array}{l}\text { †P } \\
\text { Evidência: } 6 \\
\text { †AE: } 6 \\
\text { País: Espanha } \\
\text { (Ucha Samartin } \\
\text { et al., 2013) }\end{array}$ & $\begin{array}{l}\text { Determinar o custo evitável da } \\
\text { utilização de intervenções do } \\
\text { farmacêutico em prescrições de } \\
\text { pacientes em hospital universitário }\end{array}$ & $\begin{array}{l}\text { Revisão de prescrições, orientação } \\
\text { à equipe médica e de enfermagem } \\
\text { e classificação da gravidade } \\
\text { Tipo de custo: direto - custo da diária } \\
\text { hospitalar (Xunta de Galicia, 2010) } \\
\text { Tipo de evento: eventos } \\
\text { adversos à medicação }\end{array}$ & $\begin{array}{l}\text { Pacientes: } 732 \\
\text { Intervenções: } 238 \\
\text { Custo evitado/dia de todos os } \\
\text { pacientes internados: US\$ 93.972,60 }\end{array}$ \\
\hline $\begin{array}{l}\text { †P } \\
\text { Evidência: } 6 \\
\text { †AE: } 6 \\
\text { País: Estados Unidos } \\
\text { (Kopp et al., 2007) }\end{array}$ & $\begin{array}{l}\text { Determinar o custo evitável na } \\
\text { utilização de intervenções do } \\
\text { farmacêutico em prescrições de } \\
\text { pacientes de cuidados intensivos } \\
\text { Levantamento de ПEAM e } \\
\text { classificação da gravidade e das } \\
\text { intervenções (Leape et al., 1999) }\end{array}$ & $\begin{array}{l}\text { Delineamento: } \\
\text { descritivo - } \\
\text { estudo de caso } \\
\text { Tipo de custo: direto - } \\
\text { somatório dos custos dos } \\
\text { medicamentos economizados, } \\
\text { com base no índice de preço do } \\
\text { consumidor (Bates et al., 1997) } \\
\text { Tipo de evento: eventos } \\
\text { adversos à medicação }\end{array}$ & $\begin{array}{l}\text { Intervenções: } 129 \\
\text { Custo evitado: US\$205.919,00 } \\
\text { a US\$ 280.421,00 }\end{array}$ \\
\hline $\begin{array}{l}\text { †P } \\
\text { Evidência: } 5 \\
\text { ¥AE: } 5 \\
\text { País: Estados Unidos } \\
\text { (Charles et al., 2014) }\end{array}$ & $\begin{array}{l}\text { Examinar os benefícios e as barreiras } \\
\text { à adoção de computerized physician } \\
\text { order entry (CPOE) em hospitais para } \\
\text { determinar os efeitos sobre erros } \\
\text { médicos e ПEAM e examinar os } \\
\text { custos e economias associados à } \\
\text { implementação dessa tecnologia }\end{array}$ & $\begin{array}{l}\text { Delineamento: revisão sistemática } \\
\text { que abrangeu apenas pesquisas } \\
\text { realizadas nos Estados Unidos } \\
\text { Período: } 2005 \text { a } 2014 \\
\text { Tipo de custo: variado } \\
\text { (revisão sistemática) } \\
\text { Tipo de evento: eventos } \\
\text { adversos à medicação }\end{array}$ & $\begin{array}{l}\text { Amostra: } 51 \text { artigos } \\
\text { O uso da tecnologia proporcionou } \\
\text { queda de } 12,5 \% \text { a } 90 \% \text { de } \Pi E A M \\
\text { Custo evitável/ano: de } \\
\text { US\$ } 7 \text { a } 16 \text { milhões }\end{array}$ \\
\hline
\end{tabular}




\begin{tabular}{|c|c|c|c|}
\hline $\begin{array}{l}\text { †P } \\
\text { Evidência: } 5 \\
\text { ‡AE: } 5 \\
\text { País: China } \\
\text { (Luo et al., 2017) }\end{array}$ & $\begin{array}{l}\text { Avaliar o impacto e o custo- } \\
\text {-benefício de intervenções } \\
\text { farmacêuticas clínicas sobre } \\
\text { o uso inadequado de ácido } \\
\text { sulfídrico profilático em pacientes } \\
\text { cirúrgicos hepatobiliares em } \\
\text { um hospital terciário chinês }\end{array}$ & $\begin{array}{l}\text { Delineamento: estudo } \\
\text { retroprospectivo caso-controle. } \\
\text { Realizado em dois momentos: } \\
\text { grupo controle é retrospectivo } \\
\text { (antes da intervenção farmacêutica) } \\
\text { e grupo caso é prospectivo (após } \\
\text { intervenção farmacêutica) } \\
\text { Tipo de custo: análise de custo- } \\
\text {-benefício (a relação custo- } \\
\text {-benefício foi calculada dividindo a } \\
\text { economia de custos do supressor } \\
\text { de ácido pelo custo do tempo } \\
\text { gasto pelo farmacêutico clínico) } \\
\text { Tipo de evento: erro de medicação }\end{array}$ & $\begin{array}{l}\text { Amostra: } 448 \text { pacientes ( } 218 \\
\text { controle e } 230 \text { caso) } \\
\text { Erros do grupo controle: } 546 \\
\text { Erros do grupo caso: } 279 \\
\text { Custo evitado/período } \\
\text { do estudo: US\$ 83,32 }\end{array}$ \\
\hline $\begin{array}{l}\text { १ADM } \\
\text { Evidência: } 7 \\
\text { ¥AE: } 4 \\
\text { País: Estados Unidos } \\
\text { (Letvak et al., 2012) }\end{array}$ & $\begin{array}{l}\text { Investigar em que medida a saúde } \\
\text { ocupacional de enfermeiros afeta } \\
\text { a produtividade, a qualidade } \\
\text { no atendimento e os custos }\end{array}$ & $\begin{array}{l}\text { Delineamento: } \\
\text { exploratório - } \\
\text { levantamento (survey) } \\
\text { Tipo de custo: direto - por meio } \\
\text { do modelo de regressão, criou-se } \\
\text { um coeficiente de presenteísmo } \\
\text { significativamente associado ao } \\
\text { erro de medicação multiplicado } \\
\text { pela média de salário anual da } \\
\text { categoria projetado pelo número } \\
\text { de enfermeiros/país (Karnon et } \\
\text { al., 2007; Karnon et al., 2008) } \\
\text { Tipo de evento: erro de medicação }\end{array}$ & $\begin{array}{l}\text { Amostra: } 1.171 \\
\text { Projeção de erro/ano: } 1986 \\
\text { Custo evitável/erro: US\$1.851,00 } \\
\text { Custo evitável/ano para } \\
\text { o país: US\$ } 2 \text { bilhões }\end{array}$ \\
\hline
\end{tabular}

*NE: nível de evidência; †P: prescrição; łAE: análise econômica; §EP: erro de prescrição; ПEAM: evento adverso à medicação; $₫$ ADM: administração de medicação. Fonte: Elaborado pelo autor.

Quadro 3. Distribuição da análise dos estudos sobre o custo dos erros de medicação e eventos adversos a medicamentos em mais de uma etapa da cadeia medicamentosa. São José do Rio Preto, SP, Brasil, 2018

\begin{tabular}{|c|c|c|c|}
\hline $\begin{array}{l}\text { Cadeia } \\
\text { medicamentosa e *NE }\end{array}$ & Objetivo & Método & Resultado \\
\hline $\begin{array}{l}\text { †P e } \ddagger \text { ADM } \\
\text { Evidência: } 6 \\
\text { §AE: } 6 \\
\text { País: Estados Unidos } \\
\text { (Lada \& Delgado } \\
\text { Junior, 2007) }\end{array}$ & $\begin{array}{l}\text { Avaliar a intervenção do } \\
\text { farmacêutico com a equipe } \\
\text { médica e de enfermagem e } \\
\text { mensurar o potencial de custo } \\
\text { evitável por essas intervenções } \\
\text { em unidade de emergência }\end{array}$ & $\begin{array}{l}\text { Delineamento: descritivo: } \\
\text { estudo de caso } \\
\text { Custo: direto } \\
\text { Tipo de custo: custo direto - } \\
\text { somatório do custo das medicações } \\
\text { e do trabalho do farmacêutico } \\
\text { Tipo de evento: erro de medicação }\end{array}$ & $\begin{array}{l}\text { Intervenções: } 1.393 \\
\text { ПEM: } 488 \\
\text { Custo evitável/ano: US\$ 3.089.328,00 }\end{array}$ \\
\hline $\begin{array}{l}\text { +P e } \neq A D M \\
\text { Evidência: } 6 \\
\text { §AE: } 6 \\
\text { País: Irã } \\
\text { (Gharekhani et } \\
\text { al., 2014) }\end{array}$ & $\begin{array}{l}\text { Avaliar a frequência, os tipos, a } \\
\text { significância clínica e os custos } \\
\text { diretos relacionados aos erros } \\
\text { de medicação detectados por } \\
\text { farmacêuticos clínicos em uma } \\
\text { unidade de nefrologia no Irã }\end{array}$ & $\begin{array}{l}\text { Delineamento: estudo transversal, } \\
\text { prospectivo, realizado em } 18 \text { meses } \\
\text { em uma unidade de nefrologia } \\
\text { Tipo de custo: custo direto, } \\
\text { realizado o cálculo segundo } \\
\text { uma estimativa em que se o } \\
\text { erro não fosse detectado nem } \\
\text { prevenido, quanto custaria no } \\
\text { final da internação do paciente } \\
\text { Tipo de evento: erro de medicação }\end{array}$ & $\begin{array}{l}\text { Total de pacientes: } 460 \\
\text { Pacientes com erros: } 350 \\
\text { ПEM: } 1.225 \text { (3,5 erros/paciente) } \\
\text { Custo evitável/paciente: US\$ 8,10 } \\
\text { Custo evitável/período do } \\
\text { estudo: US\$ 3.286,60 }\end{array}$ \\
\hline $\begin{array}{l}\text { †P, } 1 \text { D, } \neq \text { ADM } \\
\text { Evidência: } 6 \\
\text { §AE: } 6 \\
\text { País: México } \\
\text { (Aceves Avila } \\
\text { et al., 2011) }\end{array}$ & $\begin{array}{l}\text { Mensurar os erros de medicação } \\
\text { e os custos dos dias perdidos de } \\
\text { trabalho em pacientes ambulatoriais } \\
\text { reumatológicos no México, por meio } \\
\text { de notificação do erro de medicação }\end{array}$ & $\begin{array}{l}\text { Delineamento: exploratório } \\
\text { Estudo de campo } \\
\text { Tipo de custo: indireto - } \\
\text { calculado pela razão entre os } \\
\text { dias de trabalho perdidos x valor } \\
\text { de salário de um dia de trabalho } \\
\text { Tipo de evento: erro de medicação }\end{array}$ & $\begin{array}{l}\text { Pacientes: } 381 \\
\text { ПEM: } 127 \\
\text { Custo evitável/ano: } \\
\text { US\$ 9.041,76 }\end{array}$ \\
\hline
\end{tabular}


†P, १D, $\neq A D M$

Evidência: 6

§AE: 4

País: Estados Unidos

(Paradis et al., 2009)
Estimar os custos e o tempo de permanência associado à notificação de eventos de segurança em três hospitais, por meio de notificação do evento adverso à medicação
Delineamento descritivo

Estudo de caso múltiplo

Tipo de custo: direto - construção

de dois modelos de regressão linear

(custo e tempo de permanência)

foi atribuída a coeficiente associado

ao **EA e à diária hospitalar

Tipo de evento: evento

adverso à medicação

\section{†P, १D, $\neq A D M$}

Evidência: 6

§AE: 4

País: Estados Unidos

(Hug et al., 2012)

\section{Avaliar os eventos adversos} à medicação, o tempo de permanência e o custo evitável em seis hospitais comunitários

\section{Delineamento: descritivo -} estudo de caso múltiplo Tipo de custo: direto - somatório do custo de internação e tempo de permanência por t+DRG e custo operacional/hospital Tipo de evento: evento adverso à medicação

\section{†P, १D, $\neq A D M$}

Evidência: 6

§AE: 5

País: França

(Ranchon et al., 2011)

\section{Avaliar os erros de medicação} de antineoplásicos com ou sem interceptação do farmacêutico quanto a frequência, gravidade e custos
Delinamento: descritivo Tipo de custo: direto - custo das horas do farmacêutico Tipo de evento: erro de medicação estudo de caso
Notificações: 4.543

Tempo de permanência de internação adicional: 2.300 dias

Custo evitável/ano: US\$ 4.149.346,00

\begin{tabular}{|c|c|}
\hline $\begin{array}{l}\text { †P, १D, } \neq A D M \\
\text { Evidência: } 6 \\
\text { §AE: } 6 \\
\text { País: Estados Unidos } \\
\text { (Cohen et al., 2005) }\end{array}$ & $\begin{array}{l}\text { Avaliar o impacto de um programa } \\
\text { de segurança à medicação } \\
\text { sobre os danos causados aos } \\
\text { pacientes por łłEAM, por meio } \\
\text { de auditoria de prontuários }\end{array}$ \\
\hline
\end{tabular}

\begin{tabular}{ll}
\hline †P, १D, ¥ADM & Avaliar o impacto da revisão \\
Evidência: 6 & farmacêutica remota de \\
§AE: 6 & prescrições médicas em três \\
País: Estados Unidos & hospitais comunitários na \\
(Schneider, 2013) & Califórnia (Estados Unidos)
\end{tabular}

Delinamento: descritivo

- estudo de caso

Tipo de custo: direto - não especifica os itens custeados

Tipo de evento: eventos adversos a medicamentos

\section{Pacientes: 108.789}

\#\#EAM: 230 pacientes (sofreram um ou mais $\neq \neq$ EAM)

Custo evitável/ano: US\$ 4,6 bilhões

Delineamento: descritivo:
estudo de caso múltiplo
Tipo de custo: direto - utilizou dados
publicados em estudos anteriores
(Classen et al., 1997; Boon, 2007)
Tipo de evento: eventos
adversos à medicação

Delineamento: exploratório -

documental

Tipo de custo: direto -método de custeio micro (bottom-up) por meio de prontuário e macro (top-down) por relatórios (Otero-Lópeza et al., 2006; Alonso Hernández et al., 2002; España, 2012; Osakidtza, 2011; Xunta de Galicia, 2012)

Tipo de evento: eventos adversos à medicação
O custo médio evitável do erro de medicação ou EAM entre as pesquisas abordadas foi US\$ 617.493.770,36 (DP: 1.561.360.890) e a mediana, de US\$372.267,56, variando de US\$ 83,32 a US\$ 5.095.640.000,00.

Quanto ao custo do erro de medicação relacionado somente à etapa da prescrição médica, os estudos mostraram estratégias preventivas por meio de revisão dos itens da prescrição médica para captura dos erros de medicação, seguida de classificação da gravidade desses erros de medicação e projeção econômica dos custos evitáveis com a interceptação da farmácia clínica na cadeia medicamentosa. Outra medida preventiva apontada foi a adequação da prescrição médica por meio da análise clínica farmacêutica e do efeito econômico na redução de custos. O sistema de informação também foi utilizado como estratégia para identificar e interceptar o erro. Sua apresentação/utiliza- 


\begin{tabular}{|c|c|c|c|}
\hline $\begin{array}{l}\text { †P, } 9 \mathrm{D}, \neq A D M \\
\text { Evidência: } 5 \\
\text { §AE: } 5 \\
\text { País: Irlanda } \\
\text { (Walsh et al., 2017) }\end{array}$ & $\begin{array}{l}\text { Estabelecer o impacto econômico } \\
\text { dos erros associados a prescrição, } \\
\text { preparo, dispensação e } \\
\text { administração de medicamentos }\end{array}$ & $\begin{array}{l}\text { Delineamento: revisão sistemática } \\
\text { Período: } 2004 \text { a } 2016 \\
\text { Tipo de custo: variado } \\
\text { (revisão sistemática) } \\
\text { Tipo de evento: erro de medicação }\end{array}$ & $\begin{array}{l}\text { Amostra: } 16 \text { estudos } \\
\text { Número de erros variou } \\
\text { de } 45 \text { a } 2.356 \\
\text { Custo por erro de medicação variou } \\
\text { de US\$2,81 a US\$121.570,24 }\end{array}$ \\
\hline $\begin{array}{l}\text { †P, } 9 D \text {, } \neq A D M \\
\text { Evidência: } 4 \\
\text { §AE: } 4 \\
\text { País: Estados Unidos } \\
\text { (Choi et al., 2016) }\end{array}$ & $\begin{array}{l}\text { Estimar a incidência, tipos e causas } \\
\text { de erros de medicação, bem como } \\
\text { seus custos em ambiente hospitalar }\end{array}$ & $\begin{array}{l}\text { Delineamento: estudo retrospectivo, } \\
\text { caso-controle e multicêntrico } \\
\text { (realizado em dois hospitais) } \\
\text { Tipo de custo: método de } \\
\text { previsão reciclado e método de } \\
\text { decomposição Blinder- } \\
\text {-Oaxaca (Basu \& Rathouz, 2005; } \\
\text { Basu et al., 2006; Blinder, 1973; } \\
\text { Fairlie, 1999; Oaxaca, 1973) } \\
\text { Tipo de evento: erro de medicação }\end{array}$ & $\begin{array}{l}\text { Pacientes: } 57.554 \\
\text { Número de erros: } 470 \\
\text { Custo dos erros pelo método de } \\
\text { previsão reciclado: US\$ 8.439,00 } \\
\text { Custo dos erros pelo método de } \\
\text { decomposição Blinder- } \\
\text {-Oaxaca: US\$ 8.898,00 }\end{array}$ \\
\hline
\end{tabular}

*NE: nível de evidência; †P: prescrição; ¥ADM: administração de medicamentos; §AE: análise econômica; ПEM: erros de medicação; १D: dispensação de medicamentos; **EA: evento adverso; †+DRG: Diagnosis Related Group; łłEAM: evento adverso à medicação.

Fonte: Elaborado pelo autor.

ção, por meio de softwares, foi capaz de interceptar a maior parte dos erros de medicamentos e subsidiar a atuação da farmácia clínica, instalando barreiras de alarme em cada item da prescrição médica programada, de acordo com protocolo terapêutico médico. Dois estudos (Lampert et al., 2008; Luo et al., 2017) dessa categoria abordam a questão do uso racional de medicamentos, ou seja, prescrever o medicamento apropriado, pela via adequada, na dose adequada e com o menor custo. Dessa forma, a adequação de medicamento, via e dose proporcionou economia, respectivamente, de US\$12.555,27/ano e US\$ 83,32 no período do estudo. Houve um estudo de revisão sistemática (Charles et al., 2014) sobre o uso do Computerized Physician Order Entry (CPOE) nos Estados Unidos (EUA), onde os custos evitáveis com erros de medicação variaram de US\$ 7 a 16 milhões. Ainda, nessa etapa, os estudos analisados apontaram o custo evitável médio anual de US\$ 2.635.783,54 (DP: $5.922 .483,014)$ e mediana de US\$280.421,00, variando de US\$ 83,32 a US\$ 16.000.000,00 (Ucha Samartin et al., 2013; Lampert et al., 2008; Aldrid et al., 2009; Nerich et al., 2012; Kopp et al., 2007; Charles et al., 2014; Luo et al., 2017).

Não houve estudo que tenha tratado a etapa da dispensação de forma exclusiva. Em relação à etapa de administração de medicamentos, foi realizada, por meio de questionário, a análise da influência da saúde ocupacional de profissionais de enfermagem no atendimento referente ao erro de medicação. O custo potencialmente evitável do erro de medicação foi estimado em U\$2.000.000.000,00 (Letvak et al., 2012).

Entre as pesquisas que abordaram mais de uma etapa da cadeia medicamentosa, duas trataram da prescrição e a administração do serviço de farmácia acompanhou as prescrições e o atendimento de paciente, fazendo intervenções para prevenir erros de medicação da equipe médica e de enfermagem. O custo evitável do erro de medicação estimado variou de US\$3.286,00 a US\$ 3.089.328,00 (Gharekhani et al., 2014; Lada \& Delgado Junior, 2007).

Os trabalhos que investigaram toda a cadeia medicamentosa (prescrição, dispensação e administração) utilizaram dados secundários da literatura, a fim de estimar a quantidade de eventos adversos a medicamentos e o custo destes. Também foram acompanhados pacientes ambulatoriais por meio de aplicação de questionário, para identificar se ocorriam erros de medicação e estimar o custo potencialmente evitável de dias de trabalho perdidos por tais pacientes. Foram feitas revisões sistemáticas sobre o tema, estudo de caso controle comparando grupos com erros de medicação e sem erros. Por fim, foram mostradas estratégias preventivas para o erro de medicação por meio da farmácia clínica e acompanhamento da equipe médica (prescrição), equipe da farmácia (dispensação) e da equipe de enfermagem (administração). Comprovou-se o uso de softwares como barreira preventiva de erros. O custo médio potencialmente evitável estimado foi de US\$1.078.982.059,72 (DP 2.140.322.531), com mediana de US\$783.328,00, variando de US\$ 8.898,00 a US\$ 5.095.640.000,00 (Ranchon et al., 2011; Aceves Avila et al., 2011; Paradis et al., 2009; Hug et al., 2012; Cohen et al., 2005; Schneider, 2013; Villar, 2013; Walsh et al., 2017; Choi et al., 2016).

Quanto à classificação do tipo de evento com erro de medicação e EAM, 11 (57,9\%) estudos abordaram erros de medicação (Lampert et al., 2008; Aldrid et al., 2009; Nerich et al., 2012; Letvak et al., 2012; Lada \& Delgado Junior, 2007; Aceves-Avila et al., 2011; Ranchon et al., 2011; Luo et al., 2017; Gharekhani et al., 2014; Walsh et al., 2017; Choi et al., 2016) e os custos variaram entre US\$ 83,32 e US\$ 2 bilhões. Quanto aos EAM, outros oito (42,1\%) estudos os abordaram (Ucha-Samartin et al., 2013; Koop et al., 2007; Paradis et al., 2009; Hug et al., 2012; Cohen et al., 2005; Schneider et al., 2013; Villar, 2013; Charles et al., 2014) e seus custos variaram de US\$ 93.972,60 a US\$ 5.095.640.000,00. 


\section{Discussão}

Este estudo permitiu visualizar um panorama global da produção científica sobre o custo dos erros de medicação e/ ou EAM, além de identificar em que etapa da cadeia medicamentosa são investigados essa problemática e o nível de evidência dessas pesquisas. Dentre os 19 artigos que compuseram a amostra, o cenário principal de investigação restringiu-se a hospitais universitários, mostrando forte ligação entre a pesquisa, o ensino e a aplicação prática da pesquisa no serviço. Houve uma média de 1,6 publicação/ano, com predomínio em 2012 e 2013. Um estudo de relevância realizado em 1997 (Bates et al., 1997) tem sua metodologia de avaliação econômica aplicada a várias pesquisas na atualidade (Ranchon et al., 2011).

Nas avaliações econômicas, encontrou-se predominância do nível de evidência 6 indicando que foram realizadas de forma parcial. Por se tratar de uma avaliação parcial, esses estudos restringiram-se ao resultado, não atingindo um enfoque de custo-efetividade, custo-utilidade, custo-benefício ou custo-minimização (Joanna Briggs Institute, 2013). Dessa forma, na avaliação econômica parcial relacionada ao custo do erro de medicação ou EAM, o objetivo primordial foi identificar o erro ou evento, quantificá-lo e realizar uma intervenção para preveni-lo.

O tipo de custo mais encontrado nos estudos de economia da saúde (Tonon et al., 2008) foi o direto (84,2\%), que está relacionado a insumos (medicamentos, recursos humanos, diária hospitalar). Houve apenas uma pesquisa (5,3\%) que utilizou o custo indireto, que se refere à perda de produtividade do mercado de trabalho (dias perdidos) - que nos achados desse estudo foi projetado em US\$ 9.041,76 (Aceves Avila et al., 2011).

Observa-se que os métodos de custeio das pesquisas não são claros, nem há demonstração detalhada dos itens e valores agregados aos cálculos resultantes do custo total de cada recurso ou de cada serviço como a diária hospitalar. Esse fato compromete a comparação com outros estudos, bem como sua replicação e, certamente, justifica tamanha assimetria dos valores dos custos potencialmente evitáveis encontrados. Custo evitável pode ser definido como custos que serão eliminados se a empresa deixar de executar alguma atividade (Bruni \& Famá, 2003). A maior parte das pesquisas selecionadas neste estudo apresentou os custos potencialmente evitáveis. Então, é possível afirmar que tais custos não existiriam se a instituição de saúde conseguisse prevenir o erro de medicação ou EAM.

No Brasil, observam-se publicações sobre o erro de medicação em outras perspectivas, como incidência (Yamamoto et al., 2011), caracterização (Teixeira \& Cassiani, 2010) e prevenção (Camerini et al., 2014). Contudo, não foram encontradas pesquisas nacionais que abordassem a questão do custo dos erros de medicação nem temática que pudesse gerar impacto financeiro para o paciente, profissional, instituição e sistema de saúde.

Há um movimento na divulgação da atuação do farmacêutico no campo clínico, farmacovigilância e farmacoeconomia, em que os estudos adotam métodos que normalmente integram os seguintes passos: 1. intervenção do farmacêutico na prescrição médica; 2. análise clínica farmacêutica e/ou uso de sistema informatizado para detecção/barreira do erro de medicação ou EAM; 3. classificação da potencial gravidade/ consequência clínica para o paciente; 4. efeito econômico com projeção do custo potencialmente evitável (Ucha Samartin et al., 2013; Ranchon et al., 2011; Lampert et al., 2008; Aldrid et al., 2009; Nerich et al., 2012; Kopp et al., 2007; Aceves Avila et al., 2011; Hug et al., 2012; Schneider, 2013). Esse método descrito anteriormente possibilita demonstrar os benefícios dessa prática profissional na prevenção de riscos - erros latentes - quase erro -, em que a maioria dos eventos não resultará em dano/prejuízo ao paciente, consequentemente são ações de evitabilidade, potencializando a minimização de erros e EAM e a probabilidade em gerar custos para as instituições.

Um estudo brasileiro realizado em instituto especializado aponta que intervenções farmacêuticas diminuem, significativamente, o número de erros de medicação, destacando, ainda, que essa prática promove a qualidade do serviço e diminui custos (Nunes et al., 2008).

As pesquisas ainda apontaram que os erros de prescrição são os mais frequentes (Ucha Samartin et al., 2013; Lampert et al., 2008; Aldrid et al., 2009; Nerich et al., 2012; Kopp et al., 2007; Gharekhani et al., 2014). Corroborando esses dados (Nunes et al., 2008), o médico foi o profissional mais acionado $(71,1 \%)$ pelas intervenções farmacêuticas. Um estudo brasileiro multicêntrico tipo survey exploratório realizado com profissionais que participaram de todas as etapas da cadeia medicamentosa mostrou que o tipo de erro mais citado foi o relacionado à prescrição e à transcrição de medicamentos (Miasso et al., 2006).

Apesar de os erros de prescrição serem os mais comuns, os erros de medicação ou EAM podem ocorrer em qualquer fase da cadeia medicamentosa, sendo de prescrição, dispensação ou administração (Cassiani et al., 2010; Belela et al., 2011). Pode-se observar que a maior parte dos estudos investigou o custo do erro de medicação ou EAM nas três etapas (47,4\%), com custo que variou de US\$ 8.898,00 a US\$ 5.095.640.00,00. Porém, houve estudos que analisaram as etapas de prescrição (US\$ 83,32 a US\$ 16.000.000,00) e administração (US\$ 2.000.000.000,00) separadamente. No entanto, nenhum estudo abordou especificamente a dispensação. Haja vista que essa etapa é tão importante quanto as outras, tornam-se necessários estudos que a abordem (Teixeira \& Cassiani, 2010), pois conhecer intimamente as causas e características 
do erro é imprescindível para intervir com barreiras efetivas. A falta de pesquisas nessa etapa da cadeia medicamentosa pode estar mascarando erros que poderiam ser evitados.

O custo evitável médio do erro de medicação ou EAM entre todas as pesquisas abordadas foi de US\$617.493.770,36, variando de US\$ 83,32 a US\$ 5.095.640.000,00. Entre as pesquisas que abordaram apenas uma etapa da cadeia medicamentosa, os erros mais custosos ocorreram na etapa da administração (US\$2.000.000.000,00) (Letvak et al., 2012) e o menos custoso, na etapa da prescrição (US\$ 83,32) (Luo et al., 2008). Reforça-se novamente a importância de se pesquisar o custo do erro na etapa da dispensação isoladamente, pois não houve estudos que abordassem somente essa etapa.

As investigações de pesquisadores enfermeiros estão mais direcionadas à etapa da administração de medicamentos (Franco et al., 2010), abordando seu preparo, administração e monitoramento, possivelmente por ser uma das atividades mais frequentemente realizadas pelos profissionais de enfermagem. Contudo, não abordam os efeitos econômicos potencialmente evitáveis da atuação preventiva da equipe de enfermagem para a segurança do paciente/família, profissional ou para a instituição, tão necessário atualmente em razão da demanda/incorporação de novas tecnologias e limitação dos recursos financeiros das instituições de saúde.

Pode-se afirmar que os estudos não apresentam ou apresentam de forma resumida o método de custo utilizado, o que dificulta a análise e entendimento dessa questão.

Acredita-se que a condução deste estudo propicia a verticalização do conhecimento produzido relativo à cadeia medicamentosa sobre o erro de medicação e seus desdobramentos que ocorrem em rede para o paciente/família, profissional e instituição. Além disso, evidenciam-se lacunas do conhecimento para a prática da enfermagem, demonstrando que novos estudos precisam ser realizados para incluir as etapas de prescrição, dispensação e administração de medicamentos. Considera-se que ações devem ser estimuladas na formação docente, no ensino e na pesquisa dessa temática, especificamente estudos que se utilizem do conhecimento da economia em saúde e do método farmacoeconômico. O enfoque sobre o nível de evidência dos estudos analisados chama atenção para a necessidade de que as pesquisas futuras sigam padrões de rigor metodológico, incluindo estudos múltiplos com o potencial de aumentar a profundidade e abrangência das conclusões, diminuindo as variações nos resultados encontrados, possibilitando comparações.

Esta revisão integrativa pode embasar gestores na busca de barreiras para a prevenção do erro de medicação e EAM, investindo na segurança do paciente.

Não foram encontradas outras revisões integrativas sobre a temática, somente duas revisões sistemáticas (Walsh et al., 2017; Charles et al., 2014). Esta pesquisa avança no sentido de não restringir a busca de dados a apenas um país ou a limita a uma ferramenta de prevenção (CPOE) (Charles et al., 2014), e apresenta dados mais atuais, uma amostra maior e aplica mais de um instrumento de avaliação de nível de evidência (Walsh et al., 2017).

Este estudo apresenta limitações por buscar apenas artigos completos e não outras formas de apresentação de pesquisas. A limitação do estudo para 12 anos e três idiomas (português, inglês e espanhol) também pode ser um ponto limitante, pois mais estudos fora do período e em outros idiomas podem ser encontrados.

\section{Conclusão}

Em geral, as pesquisas que fizeram parte da amostra do estudo não abordam a problemática do custo do erro de medicação e EAM em sua completude. Há predominância de estudos que fazem estimativas de custos evitáveis e em sua maioria com nível 6 (baixo) de evidência. Quanto à etapa da cadeia medicamentosa pesquisada, houve predomínio em trabalhar com toda a cadeia medicamentosa, ou seja, desde a prescrição até a administração dos medicamentos. No entanto, pode-se concluir que os erros de medicação e EAM são dispendiosos para o sistema de saúde.

Outro aspecto é que os estudos na perspectiva da economia da saúde mencionam a classificação do tipo de custo utilizado, mas não apresentam ou apresentam de forma resumida o método de custo utilizado, o que dificulta a análise e o entendimento dessa questão. São necessários mais estudos com métodos aprimorados e melhores níveis de evidências.

Não foram identificadas pesquisas nacionais sobre a temática. Dessa forma, não há como afirmar que esta também é uma realidade brasileira. Com esses dados, pode-se afirmar que são importantes novos estudos, com métodos mais apurados para quantificar esse desperdício a fim de melhorar a segurança do paciente, a questão econômica e incentivar a implantação de tecnologias para prevenir erros de medicação e EAM de forma consciente.

\section{Referências bibliográficas}

Aceves-Avila FJ, Benites-Godinez V, Ramus-Remus C. Cost of medication errors in rheumatic patients in Mexico. Clin Rheumatol. 2011;30(11):1421-4.

Aldrid VE, Park HK, Bounthavong M, Morreale AP. Inplementing a comprehensive, 24-hour emergency department pharmacy program. Am J Health Syst Pharm. 2009;66(21):1943-7.

Alonso-Hernández P, Otero-Lópeza MJ, Maderuelo-Fernandez JA. Ingresos hospitalarios causados por medicamentos: incidencia, características y coste. Farm Hosp [Internet]. 2002;26(2):77-89. Disponível em: http://www. elsevier.es/es-revista-farmacia-hospitalaria-121-articulo-ingresos-hospitalarioscausados-por-medicamentos-13118908. Acesso em: 2 ago 2016.

Anacleto TA, Rosa MB, Neiva HM, Martins MAP. Erros de medicação. Farmacovigilância hospitalar: como implantar. Pharmacia Bras [Internet]; 2010: Disponível em: http://www.cff.org.br/sistemas/geral/revista/ pdf/124/encarte_farmaciahospitalar.pdf. Acesso em: 29 jul. 2016. 
Basu A, Arondekar BV, Rathouz PJ. Scale of interest versus scale of estimation: comparing alternative estimator for incremental costs of a comorbidity. Health Econ. 2006;15:1091-107.

Basu A, Rathouz PJ. Estigmating marginal and incremental effects on health outcomes using flexible link and variance function models. Biostatistics. 2005;6:93-109.

Bates DW, Spell N, Cullen D, Burdick E, Laird N, Petersen LA, et al. The costs of adverse drugs events in hospitalized patients. JAMA [Internet]. 1997;277(4). Disponível em: http://jama.jamanetwork.com/article. aspx?articleid=413545. Acesso em: 30 jul 2016.

Belela ASC, Peterlini MAS, Pedreira MLG. Erros de medicação: definições e estratégias de prevenção. São Paulo: Coren [Internet]; 2011. Disponível em: http://inter.corensp.gov.br/sites/default/files/erros_de_ medicacaodefinicoes_e_estrategias_de_prevencao.pdf. Acesso em: 25 jul 2016.

Blinder AS. Wage discrimination: reduces form and structural variable. J Hum Resour. 1973;8:436-55.

Boon AD. Telepharmacy at a critical access hospital. Am J Health Syst Pharm. 2007;64:242-4.

Bruni AL, Famá R. Gestão de custos e formação de preços com aplicações na calculadora HP 12C e Excel. São Paulo: Atlas; 2003.

Camerini FG, Silva LD, Mira AJM. Ações de enfermagem para administração segura de medicamentos: uma revisão integrativa. Rev Pesqui Cuid Fundam (Online) [Internet]. 2014;6(4):1655-65. Disponível em: www. seer.unirio.br/index.php/cuidadofundamental/article/download/2081/ pdf_1213. Acesso em: 12 jan 2017.

Cassiani SHB, Monzani AAS, Silva AEBC, Fakih FT, Opitz SP, Teixeira TCA. Identificación y análisis de los errores de medicación en seis hospitales brasileños. Cienc Enferm [Internet]. 2010;16(1):85-95. Disponível em: http://www.scielo.cl/scielo.php?script=sci_arttext\&pid =S0717-95532010000100010. Acesso em: 25 jul 2016.

Charles K, Cannon M, Hall R, Coustasse A. Can utilizing a Computerized Provider Order Entry (CPOE) system prevent hospital medical errors and adverse drug events? Perspectives in Health Information Management [Internet]. 2014;11(fall):1-16. Disponível em: https://www.ncbi.nlm.nih. gov/pmc/articles/PMC4272436/. Acesso em: 25 jun 2018.

Choi I, Lee S, Flynn L, Kim C, Lee S, Kim NK, et al. Incidence and treatment costs attributable to medication errors in hospitalized patients. Res Social Adm Pharm. 2016;12(3):428-37.

Classen DC, Pestotnik SL, Evans S, Lloyd JF, Burke JP. Adverse drug events in hospitalized patients: excess length of stay, extra costs, and attributable mortality. JAMA. 1997;277(4):301-6.

Cohen MM, Kimmel NL, Benage MK, Cox MJ, Sanders N, Spence D, et al. Medication safety program reduces adverse drugs events in a community hospital. Qual Saf Health Care. 2005;14(3):169-74.

España. Ministerio de Sanidad, Servicios Sociales e Igualdad. Sistema Nacional de Salud SNS España [Internet]; 2012. Disponível em: http:// www.msssi.gob.es/organizacion/sns/libroSNS.htm. Acesso em: 13 nov 2016.

Fairlie RW. The absence of the African-American owned business: an analysis of the dynamics of self-employment. J Labor Econ. 1999;17:80-108.

Franco JN, Ribeiro G, D'Innocenzo M, Barros BPM. Percepção da equipe de enfermagem sobre fatores causais de erros na administração de medicamentos. Rev Bras Enferm [Internet]. 2010;63(6):927-32. Disponível em: http://www.scielo.br/scielo.php?script=sci_arttext\&pid $=$ S0034-71672010000600009. Acesso em: 12 jan 2017.

Gharekhani A, Kanani N, Khalili H, Dashti-Khavidaki S. Frequency, types, and direct related costs of medication errors in an academic nephrology ward in Iran. Ren Fail. 2014; 36(8):1268-72.
Hug BL, Keohane C, Seger DL, Yoon C, Bates DW. The costs of adverse drugs events in community hospitals. Jt Comm J Qual Patient Saf. 2012;38(3):120-6.

Joanna Briggs Institute. Levels of evidence for economic evaluation. New JBI levels of evidence [Internet]. 2013. Disponível em: http://joannabriggs. org/assets/docs/approach/JBl-Levels-of-evidence_2014.pdf. Acesso em: 12 ago 2016.

Kane-Gill SL, Jacobi J, Rothschild JM. Adverse drug events in intensive care units: risk factors, impact, and the role of team care. Crit Care Med. 2010;38(6):83-9.

Karnon J, Mclntosh A, Dean J, Bath P, Hutchinson A, Oakley J, et al. A prospective hazard and improvement analytic approach to prediting the effectiveness of medication error interventions. Saf Sci. 2007;45(4):523-39.

Karnon J, McIntosh A, Dean J, Bath P, Hutchinson A, Oakley J, et al. Modelling the expected net benefits of intervention to reduce the burden of medication errors. J Health Serv Res Policy. 2008;13(2):85-91.

Kohn LT, Corrigan JM, Donaldson ME. To err is human: building a safer health system. Washington: National Academy Press [Internet]; 1999. Disponível em: https://www.ncbi.nlm.nih.gov/books/NBK225182/. Acesso em: 12 jun 2016.

Kopp BJ, Mrsan M, Erstad BL, Duby JJ. Cost implications of and potential adverse events prevented by intervention of a critical care pharmacist. Am J Health Syst Pharm. 2007;64(23):2483-7.

Lada P, Delgado Junior G. Documentation of pharmacists' interventions in an emergency department and associated cost avoidance. Am J Health Syst Pharm. 2007;64(1):63-8.

Lampert ML, Kraehenbuehl S, Hug BL. Drug-related problems: evaluation of a classification system in the daily practice of a Swiss University Hospital. Pharm Worls Sci [Internet]. 2008;30(6):768-76. Disponível em: http://link.springer.com/article/10.1007\%2Fs11096-008-9213-8. Acesso em: 21 jul 2016.

Leape LL, Cullen DJ, Clapp MD, Burdick E, Demonaco HJ, Erickson JI, et al. Pharmacist participation on physician rounds and adverse drug events in the intensive care unit. JAMA. 1999;282(3):267-70.

Lee AJ, Boro MS, Knapp KK, Meier JL, Korman NE. Clinical and economic outcomes of pharmacist recommendations in a Veterans Affairs medical center. Am J Health Syst Pharm. 2002;59(21):2070-7.

Letvak SA, Ruhm CJ, Gupta SN. Nurses' presenteeism and its effects on self-reported quality of care and costs. AJN. 2012;112(2):30-8.

Luo H, Fan Q, Xiao S, Chen K. Impact of clinical pharmacist interventions on inappropriate prophylactic acid suppressant use in hepatobiliary surgical patients undergoing elective operations. PLoS One. [Internet] 2017;12(10):1-15. Disponível em: http://journals.plos.org/plosone/ article?id=10.1371/journal.pone.0186302. Acesso em: 25 jun 2018.

Melnyk BM, Fineout-Overholt E. Evidence-based in nursing and healthcare 2a ed. Filadélfia: Lippincott Williams \& Wilkins [Internet]; 2011. Disponível em: http://file.zums.ac.ir/ebook/208-Evidence-Based\%20Practice\%20 in\%20Nursing\%20\&\%20Healthcare\%20-\%20A\%20Guide\%20to\%20 Best\%20Practice,\%20Second\%20Edition-Be.pdf. Acesso em: 25 jul 2016.

Miasso Al, Grou CR, Cassiani SHB, Silva AEBC, Fakih FT. Erros de medicação: tipos, fatores causais e providências tomadas em quatro hospitais brasileiros. Rev Esc Enferm USP [Internet]. 2006;40(4):524-32. Disponível em: http://www.scielo.br/scielo.php?script=sci_arttext\&pid $=$ S0080-62342006000400011. Acesso em: 12 jan 2017.

Nerich V, Borg C, Villanueva C, Thiery-Vuillemin A, Helias P, Rohrlich PS, et al. Economic impact of prescribing error prevention with computerized physician order entry of injectable antineoplastic drugs. J Oncol Pharm Pract. 2012;19(1):8-17. 
Nunes PHC, Pereira BMG, Nominato JCS, Albulquerque EM, Silva LFN, Castro IRS, et al. Intervenção farmacêutica e prevenção de eventos adversos. Rev Bras Ciênc Farm [Internet]. 2008;44(4):691-9. Disponível em: http:// www.scielo.br/pdf/rbcf/v44n4/v44n4a16.pdf. Acesso em: 12 jan 2017.

Oaxaca R. Male-female wage differentials in urban labor markets. Int Econ Rev. 1973;14:693-709.

Osakidetza. Tarifas para facturación de servicios sanitarios y docentes de Osakidetza para 2012. Vitoria-Gasteiz [Internet]; 2011. Disponível em: http://www.osakidetza.euskadi.net/r85ekgnrl00/es/contenidos/ informacion/libro_tarifas/es_libro/adjuntos/tarifas2012.pdf. Acesso em: 13 nov 2016.

Otero-Lópeza MJ, Alonso-Hernández P, Maderuelo-Fernandez JA, Garrido-Corroa B, Domínguez-Gila A, Sánchez-Rodríguez A. Acontecimentos adversos prevenibles causados por medicamentos em pacientes hospitalizados. Med Clin (Barc) [Internet]. 2006;126(3):81-7. Disponivel em: http://www.ismpespana.org/ficheros/Fichero21.pdf. Acesso em: 2 ago 2016.

Paradis AR, Stewart VT, Bayley KB, Brown A, Bennett AJ. Excess cost and length of stay associated with voluntary patient safety events reports in hospitals. Am J Med Qual. 2009;24(1):53-60.

Pazin-Filho A, Frezza G, Matsuno AK, Alcântara ST, Cassiolato S, Bitar JPS, et al. Princípios de prescrição médica hospitalar para estudantes de medicina. Medicina (Ribeirão Preto) [Internet]. 2013;46(2):183-94. Disponível em: http://revista.fmrp.usp.br/2013/vo|46n2/TEM_Princ\%EDpios\%20 de\%20Prescri\%E7\%E30\%20M\%E9dica\%20Hospitalar\%20para\%20 Estudantes\%20de\%20Medicina.pdf. Acesso em: 25 jul 2016.

Ranchon F, Salles G, Späth HM, Schwiertz V, Vantard N, Parat S, et al. Chemotherapeutic errors in hospitalized cancer patients: attributable damage and extra costs. BMC Cancer [Internet]. 2011;11:478.

Disponivel em: http://scielo.isciii.es/scielo.php?script=sci_arttext\&pid $=$ S1130-63432013000100009 doi: 10.1186/1471-2407-11-478. Acesso em: 25 jul 2016.

Schneider PJ. Evaluating the impact of telepharmacy. Am J Health Syst Pharm. 2013;70(23):2130-5.

Teixeira TCA, Cassiani SHB. Análise da causa raiz: avaliação de erros de medicação em um hospital universitário. Rev Esc Enferm USP [Internet]. 2010;4(1). Disponível em: http://www.scielo.br/scielo. php?script=sci_art text\&pid=S0080-62342010000100020. Acesso em: 20 jan. 2017.
Tonon LM, Tomo TT, Secoli SR. Farmacoeconomia: análise de uma perspectiva inovadora na prática clínica da enfermeira. Texto Contexto Enferm [Internet]. 2008;17(1):177-82. Disponível em: http://www.scielo.br/ scielo.php?script=sci_arttext\&pid=S0104-07072008000100020. Acesso em: 20 jul 2016.

Ucha-Samartin M, Pichel-Loureiro A, Vázquez-López C, Payero MA, Parente DP, Castro NML. Impacto económico de la resolución de problemas relacionados con medicamentos en un servicio de urgencia. Farm Hosp. 2013;37(1):59-64.

Villar FA. Aproximación a los costs de la no seguridad en el Sistema Nacional De Salud. Rev Esp Salud Publica [Internet]. 2013;87(3):283-92. Disponível em: http://scielo.isciii.es/scielo.php?script=sci_arttext\&pid =S1135-57272013000300008. Acesso em: 2 ago 2016.

Walsh EK, Hansen CR, Sahm LJ, Kearney PM, Doherty E, Bradley CP. Economic impact of medication error: a systematic review. Pharmacoepidemiol Drug Saf. 2017;26(5):481-97.

Whittemore R, Chao A, Jang M, Minges KE, Park C. Methods for knowledge synthesis: an overview. Heart Lung [Internet]. 2014;43(5):453-61. Disponível em: http://www.heartandlung.org/article/S01479563(14)00189-7/fulltext. Acesso em: 28 jul 2016.

Xunta de Galicia. Decreto 221/2012 por el que se establecen las tarifas de los servicios sanitários prestados en los centros dependientes del Servicio Gallego de Salud y en las fundaciones públicas sanitarias. Diario Oficil de Galicia [Internet]. 2012;(222):43675. Disponível em: https://www. xunta.gal/dog/Publicados/2012/20121121/AnuncioC3K1-091112-0002_ es.html. Acesso em: 8 ago 2016.

Xunta de Galicia. Real Decreto 160/2010, del 23 de septiembre de 2010, por el que se establecen las tarifas de los servicios sanitários prestados em los centros dependientes del Servizo Galego de Saúde y em las fundaciones públicas sanitárias. Diario Oficil de Galicia [Internet]. 2010;(188):16534. Disponível em: http://www.xunta.gal/dog/Publicados/2010/20100929/ Anuncio2E44A_es.html. Acesso em: 8 ago 2016.

Yamamoto MS, Peterlini MAS, Bohomol E. Notificação espontânea de erros de medicação em hospital universitário pediátrico. Acta Paul Enferm [Internet]. 2011;24(6):766-71. Disponível em: http://www.scielo.br/scielo. php?script=sci_arttext\&pid=S0103-21002011000600006. Acesso em: 2 ago 2016. 